\title{
Middle cerebral artery stroke following massive hornet sting: a case report
}

\author{
Gopal Sedain ${ }^{1}$, Gentle Sunder Shrestha ${ }^{2}$ \\ 1 Neurosurgery, Alka Hospital, Jawalakhel, Lalitpur, NEPAL \\ 2 Anaesthesiologist and Critical Care Physician, Alka Hospital, \\ Jawalakhel, Lalitpur, NEPAL
}

\begin{abstract}
Hornet stings are frequently encountered in practice in Nepal. Majority of patients sustain minor illness. However, complications like anaphylactic shock and the rare acute kidney injury, multiple organ dysfunction and acute myocardial infarction have been reported. Ischemic stroke following hornet stings has been reported infrequently in scientific publications. We report a case of fatal right Middle Cerebral artery territory ischemic stroke and acute kidney injury in a 40-year farmer.
\end{abstract}

\section{INTRODUCTION}

Human encounter with hornets has been found in plantation, cultivation and forest areas. They are aggressive upon disturbance and attack in swarms causing victims to sustain multiple stings. The sting is excruciatingly painful and the insect leaves the stinger. Unlike typical bees, hornets and wasps do not die after stinging because their stingers are not barbed and are not pulled out of their bodies. Hornet stings are more painful to humans than typical wasp stings because hornet venom contains a large amount (5\%) of acetylcholine.

Hornets, like many social wasps, can mobilize the entire nest to sting in defense, which is highly dangerous to animals and humans. The attack pheromone is released in case of threat to the nest.

The majority of sufferers recover with minor illness. But fatal and non-fatal complications that arise from such stings include anaphylaxis, acute kidney injury (8), multiple organ dysfunction (7), myocardial infarction (3) and ischemic stroke $(1,2,5,6)$. Vasoactive, inflammatory, and thrombogenic peptides and amines, including histamine, leukotrienes, and thromboxane are responsible for the end organ complications. The allergenic proteins such as phospholipases which elicit IgE responses, resulting in mast cell activation, underlie the anaphylaxis.

\section{CASE REPORT}

A 40-year-old patient was referred to the emergency department for decreased consciousness after sting by a swarm of hornets. The

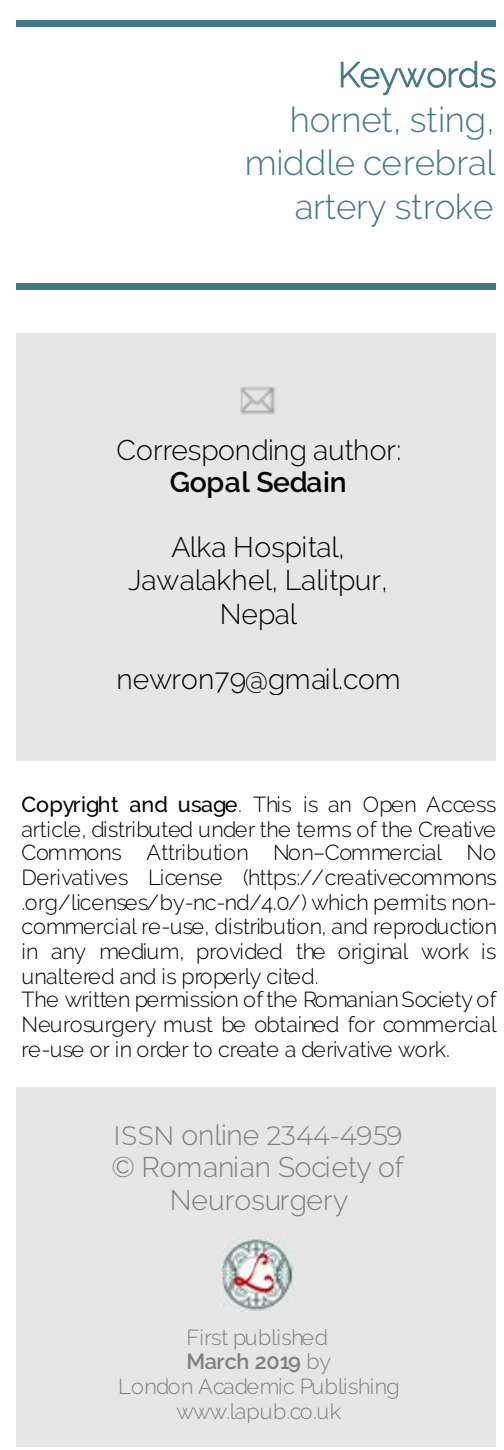


previously healthy farmer from the Dang valley in western Nepal was stung by around 100 hornets when he was collecting maize crops in his field. He was immediately taken to local hospital where he received chlorpheniramine (antihistamine) and hydrocortisone. The patient had fall in sensorium for which he was referred to our center. The family's medical history did not reveal thromboembolic disease or significant neurological and autoimmune rheumatoid disease. Upon arrival, his GCS was poor (6/15). Physical examination revealed multiple stings

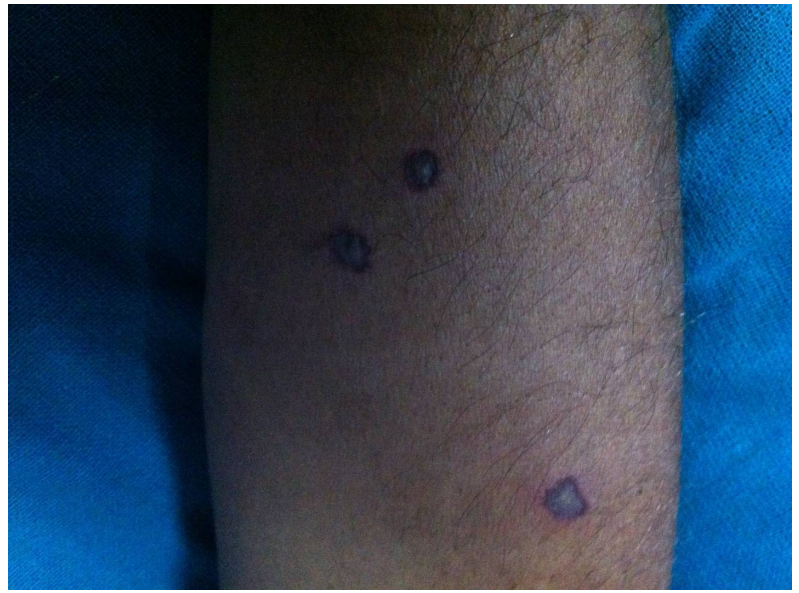

FIGURE 1: Hornet sting induced local erythematous changes in the forearm.

FIGURE 2: Plain CT head revealing large right MCA infarct with mass effect

\section{DISCUSSION}

Hornet venom contains vasoactive, inflammatory, and thrombogenic peptides and amines, including histamine, leukotrienes, and thromboxane. The venom also contains allergenic proteins such as phospholipases which elicit an IgE response, resulting in mast cell activation which is the hallmark of anaphylaxis.

Pain, wheal, flare, edema and swelling, which are generally self-limiting, constitute local disease. Multiple stings can lead to vomiting, diarrhea, generalized edema, dyspnea, and hypotension.

Severe systemic complications include anaphylaxis which constitutes the leading cause of death, acute kidney injury, multiple organ dysfunctions, disseminated intravascular coagulation, myocardial infarction, Rhabdomyolysis and neurological disease.

Numerous neurological complications include ischemic stroke, venous sinus thrombosis, ocular in his body including his face and scalp. (Fig 1) He had acute kidney injury (creatinine level $9.5 \mathrm{mg} \%$ ). His serum LDH was 5211(normal range: 225-450) and CPK of 3652 (normal <170U/L). He had severe metabolic acidosis with Bicarbonate level of $15 \mathrm{meq} / \mathrm{l}$. His CT head revealed a large MCA (middle cerebral artery) ischemic stroke with mass effect and midline shift. (Fig 2) He underwent dialysis for acute kidney injury, however patient succumbed to his illness.

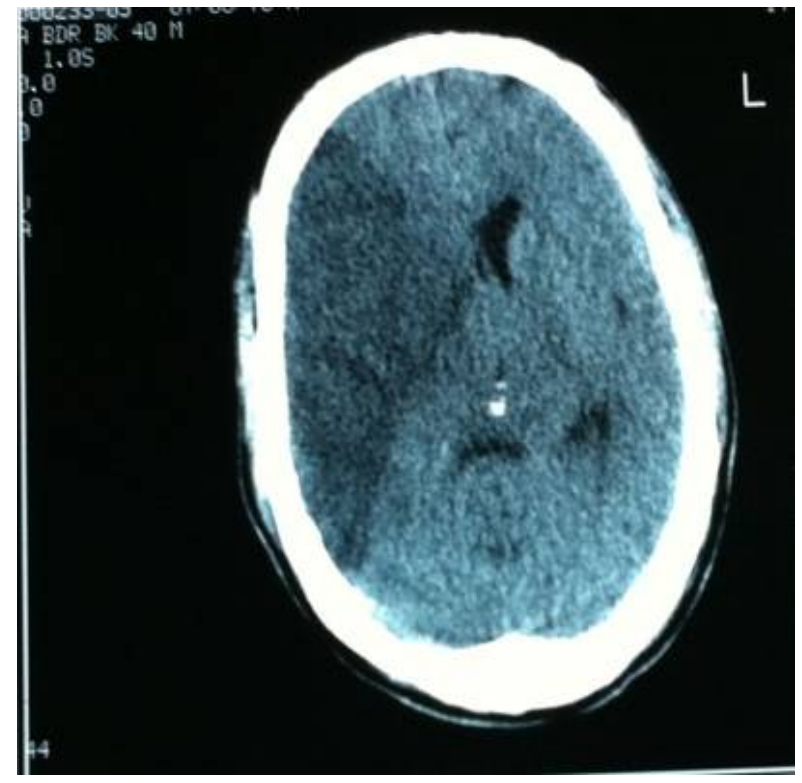

myasthenia gravis and thrombotic thrombocytopenic purpura. The proposed underlying mechanism of early ischemic stroke includes hypotension of anaphylaxis and vasospasm due to treatment with adrenaline. The delayed phenomena leading to infarctions appear to be spasms caused by vasoactive substances and thrombosis induced by thrombogenic factors in venom (1).

The other neurological complications of stings which have been reported are individual case reports of ocular myasthenia gravis, optic neuritis, limb numbness, trigeminal neuralgia and encephalopathy. Postulated mechanisms include both toxic effect of venom and hypersensitivity to venom (6).

In the case of acute myocardial infarction following hornet stings, the postulated mechanism is a combination of coronary vasoconstriction and platelet aggregation secondary to mediators released after wasp sting, aggravated by exogenous adrenaline given as part of the treatment. 
In our patient, we postulate that the systemic immune mediated reaction to the bee sting caused vasoconstriction and a prothrombotic state with subsequent ischaemia leading to stroke.

\section{CONCLUSIONS}

Insect stings can lead to neurological complications and can be fatal. Timely intervention is required to prevent these.

\section{REFERENCES}

1. Bhat $R$, Bhat $K R$, Pais R.Bilateral haemorrhagic cerebellar infarction following honeybee sting. J Assoc Physicians India 2002; 50:721-2.

2. Day JM. Death due to cerebral infarction after wasp stings. Arch Neurol 1962; 7:184-186
3. Levine HD. Acute myocardial infarction following wasp sting. Report of two cases and critical survey of the literature. Am HeartJ 1976 ;91: 365-374

4. Paudel B, Paudel K. A study of wasp bites in a tertiary hospital of western Nepal. Nepal Med Coll J 2009;11: 52-56.

5. Riggs JE, Ketonen LM, Bodensteiner JB, Benesch CG. Wasp sting-associated cerebral infarction: a role for cerebrovascular sympathetic innervation. Clin Neuropharmacol 1993; 16: 362-365.

6. Sachdev A, Mahapatra M, D'Cruz S, Kumar A, Singh R. Wasp sting induced neurological manifestations. Neurol India $2002 ; 50: 319-321$

7. Watemberg N, Weizman Z, Shahak E, Aviram M, Maor E. Fatal multiple organ failure following massive hornet stings. J Toxicol Clin Toxicol 1995 ;33: 471-474.

8. Xuan BH, Mai HL, Thi TX, Thi MT, Nguyen HN. Swarming hornet attacks: shock and acute kidney injury--a large case series from Vietnam. Nephrol Dial Transplant $2010 ; 25$ : $1146-1150$ 